\title{
Implementation of the Actuator Cylinder Flow Model in the HAWC2 code for Aeroelastic Simulations on Vertical Axis Wind Turbines
}

\author{
Aagaard Madsen, Helge; Larsen, Torben J.; Schmidt Paulsen, Uwe; Vita, Luca
}

Published in:

Proceedings of 51st AIAA Aerospace Sciences Meeting including the New Horizons Forum and Aerospace Exposition

Publication date:

2013

Link back to DTU Orbit

Citation (APA):

Aagaard Madsen , H., Larsen, T. J., Schmidt Paulsen, U., \& Vita, L. (2013). Implementation of the Actuator Cylinder Flow Model in the HAWC2 code for Aeroelastic Simulations on Vertical Axis Wind Turbines. In Proceedings of 51 st AlAA Aerospace Sciences Meeting including the New Horizons Forum and Aerospace Exposition AIAA.

\section{General rights}

Copyright and moral rights for the publications made accessible in the public portal are retained by the authors and/or other copyright owners and it is a condition of accessing publications that users recognise and abide by the legal requirements associated with these rights.

- Users may download and print one copy of any publication from the public portal for the purpose of private study or research.

- You may not further distribute the material or use it for any profit-making activity or commercial gain

- You may freely distribute the URL identifying the publication in the public portal 


\title{
Implementation of the Actuator Cylinder Flow Model in the HAWC2 code for Aeroelastic Simulations on Vertical Axis Wind Turbines
}

\author{
H. Aa. Madsen ${ }^{1}$, T. J. Larsen ${ }^{2}$, U. S. Paulsen ${ }^{3}$, L. Vita ${ }^{4}$ \\ Technical University of Denmark, Department of Wind Energy \\ Campus Risoe, DK 4000 Roskilde Denmark
}

\begin{abstract}
The paper presents the implementation of the Actuator Cylinder (AC) flow model in the HAWC2 aeroelastic code originally developed for simulation of Horizontal Axis Wind Turbine (HAWT) aeroelasticity. This is done within the DeepWind project where the main objective is to explore the competitiveness of VAWTs for floating MW concepts. The AC model is a 2D flow model and has thus some advantages compared with the stream tube models often used in VAWT aerodynamic and aeroelastic simulation models. A major finding presented in the present paper is a simple way to correct the results from the linear version of the AC model so that they correlate closely with the results of the full AC model. The linear model has very low computational requirements and is thus well suited for implementation in an aeroelastic model where the induction in a number of points on the rotor swept surface is updated at each time step. The AC model is described and the implementation of the model in HAWC2 is briefly presented. Results illustrating the accuracy of the different versions of the AC model are presented. Finally, initial simulations on the 5MW baseline rotor with the new HAWC2 version with the AC model implemented are presented.
\end{abstract}

\section{Nomenclature}

$\begin{array}{ll}a & =\text { axial induction factor } \\ a_{\text {lin }} & =\text { axial induction factor from linearized flow model } \\ B & =\text { number of blades } \\ C_{p} & =\text { rotor power coefficient } \\ C_{T} & =\text { rotor thrust coefficient } \\ D & =\text { drag force per unit blade length } \\ f_{x} & =\text { volume force in stream wise direction } \\ f_{y} & =\text { volume force in lateral direction } \\ F_{n} & =\text { aerodynamic blade force perpendicular to blade chord } \\ F_{t} & =\text { aerodynamic blade force parallel to blade chord } \\ g_{x} & =\text { induced or second order force in stream wise direction } \\ g_{x} & =\text { induced or second order force in stream wise direction } \\ i & =\text { azimuth index } \\ j & =\text { azimuth index } \\ k_{a} & =\text { induction amplification factor }\end{array}$

\footnotetext{
${ }^{1}$ Research Specialist, DTU Wind Energy, Campus Risoe, Frederiksborgvej 399, DK 4000 Roskilde

${ }^{2}$ Senior Researcher, DTU Wind Energy, Campus Risoe, Frederiksborgvej 399, DK 4000 Roskilde

${ }^{3}$ Senior Researcher, DTU Wind Energy, Campus Risoe, Frederiksborgvej 399, DK 4000 Roskilde

${ }^{4}$ Researcher, DTU Wind Energy, Campus Risoe, Frederiksborgvej 399, DK 4000 Roskilde
} 


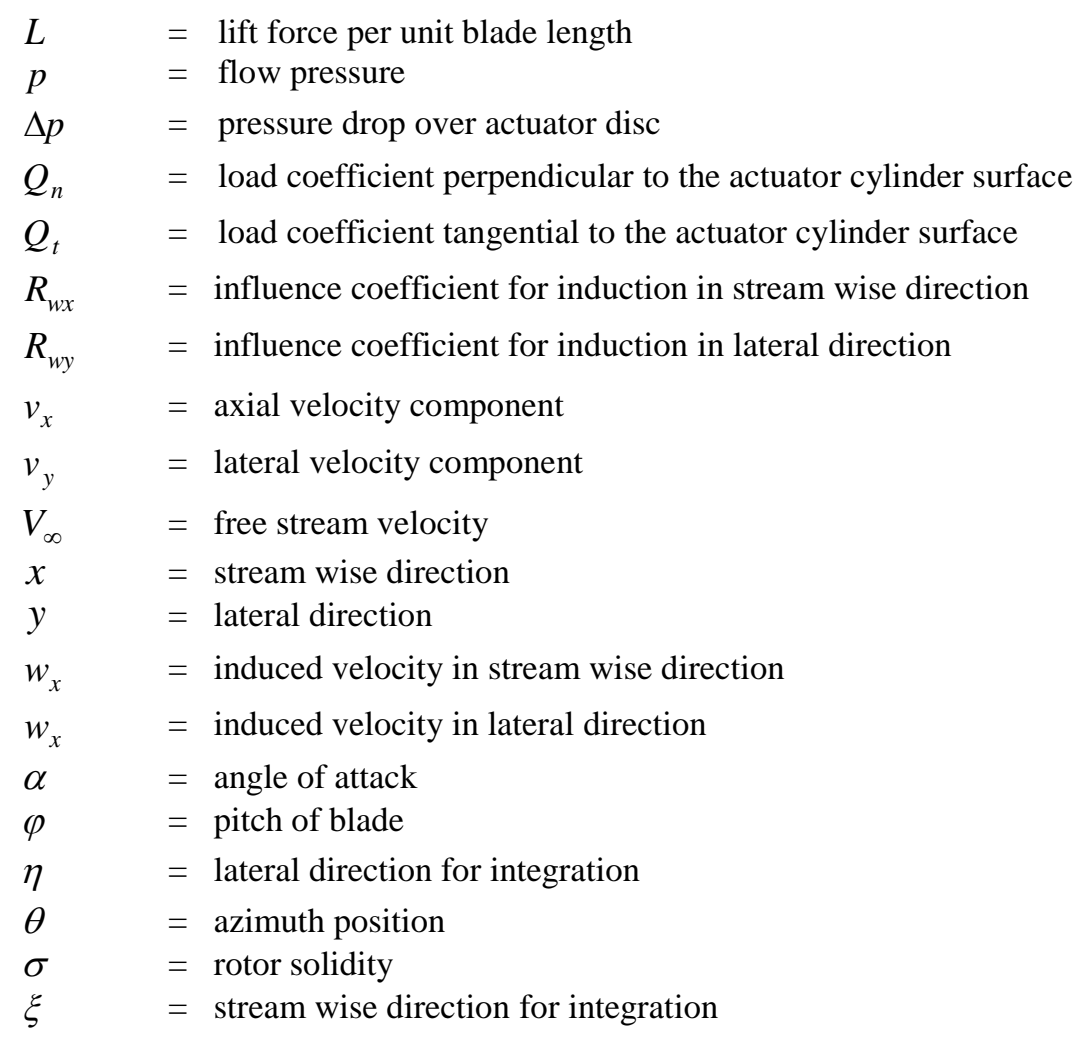

\section{Abreviations}

$\mathrm{AD} \quad=$ actuator disc

$\mathrm{AC} \quad=$ actuator cylinder

HAWT $=$ horizontal axis wind turbine

VAWT $=$ vertical axis wind turbine

MOD LIN $=$ modified linear model

\section{Introduction}

considerable part of the future wind turbine installations will be erected offshore and for sites deeper than about $50 \mathrm{~m}$, the floating platforms are more competitive than the mono piles used for shallow waters. For these floating concepts the VAWT turbines have recently been reconsidered for use in the MW size for several reasons. One major advantage is the low center of gravity of the rotor and another is the independence of rotor aerodynamic performance on wind direction.

In the DeepWind project ${ }^{1,2}$ a strongly simplified 5MW, floating VAWT is being developed where the water is used as bearing for the rotating floater extending directly from the main shaft as shown in Fig. 1a. The concept features a long rotating spar buoy as support structure, direct drive subsea generator, torque absorption and mooring cables which are anchored into the sea bed, Fig. $1 \mathrm{~b}$.

The combined aeroelastic/hydrodynamic analysis of such a design is challenging. It requires a numerical tool that at the same time can model the turbulent aerodynamic loading on the rotor blades, the wave loading on the rotating shaft, the buoyancy on the submerged, rotating shaft, the Magnus force loading on the rotating shaft and the mooring line dynamics and forces. Although the aeroelastic code $\mathrm{HAWC}^{3}$ from the beginning was developed for simulations on horizontal axis wind turbines HAWT's, most parts of the model are written so general that it directly can be used for VAWT's. This is not least due to the multi-body concept used for the structural dynamics modeling. However, also the wave loading, the buoyancy and the mooring line dynamics have only required minor extensions in order to model the concept. Also the aerodynamic blade loading modeling is so general that it directly can model the VAWT blade loads but not the induction. Finally, the Magnus forces occurring on the rotating shaft had to be added for sea water current conditions. 


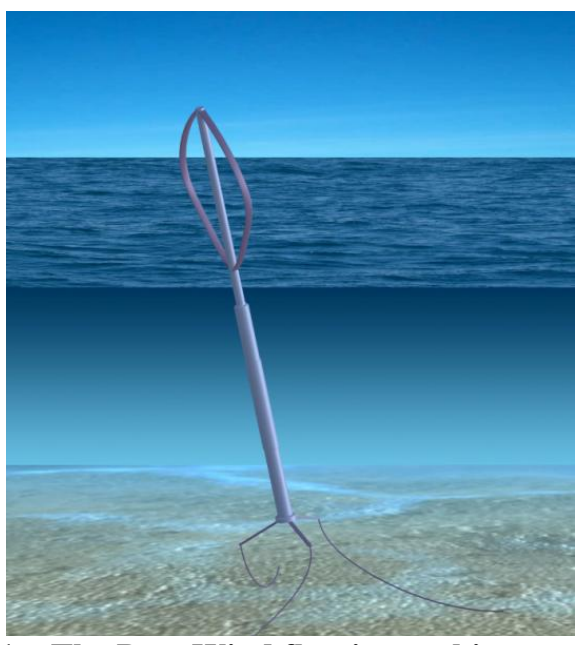

Figure 1a. The DeepWind floating turbine concept.

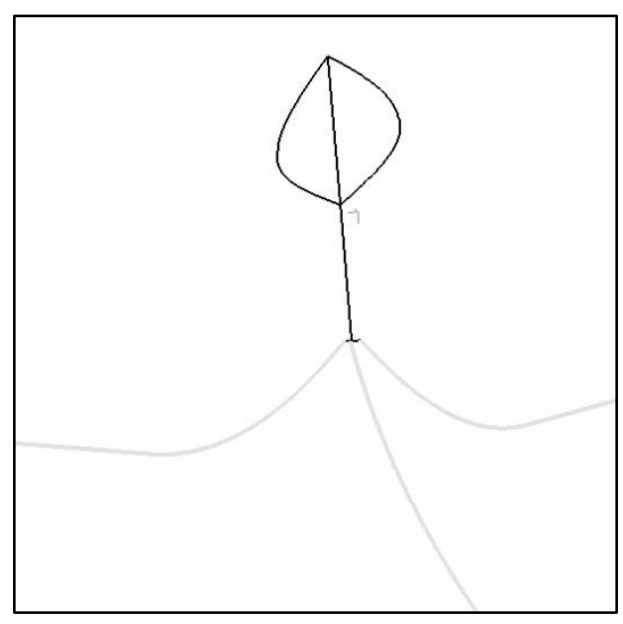

Figure 1b. A 5MW DeepWind turbine concept with mooring lines modeled in HAWC2. Rotor height is $130 \mathrm{~m}$ and the rotating floater is $107 \mathrm{~m}$ long.

During a recent $\mathrm{PhD}$ study ${ }^{4}$ the modeling of induction and Magnus force was implemented using the DLL interface capability of HAWC2 and the first aeroelastic simulations on a 2MW and 5MW DeepWind prototype were performed. Further results on aeroelastic simulations on the 5MW turbine have been presented in Ref. 5 .

The modeling presented in Ref. 4 did not utilize the full build in capabilities in HAWC2 such as the dynamic stall modeling and the turbulent inflow modeling as the aerodynamic loading was computed in a DLL outside HAWC2. Therefore, the objective of the present paper is the presentation of an aerodynamic VAWT induction model well suited for integration in an aeroelastic model due to its low computational requirements. The implementation of the induction model in HAWC2 is briefly presented as well as an example of simulations of turbulent inflow on a 5MW turbine. A more detailed description of the implementation can be found in Ref. 6 .

In the past the so called multiple stream tube MST model by Strickland ${ }^{7}$ and the double multiple stream tube DMST model by Paravischivoiu ${ }^{8}$ have been widely used for aerodynamic analyses. For computations of aeroelastic response, the TRES4D code from Sandia National Laboratories ${ }^{9}$, has been used where the induction is computed with the DMST model. The MST and the DMST models have their origin from the very common BEM model used for HAWT's and are popular due to their low computing requirements necessary in aeroelastic simulations where the induction has to be updated at each time step. However, this type of models has also some build in drawbacks; they are one-dimensional and they are modeling the flow through a plane actuator disc (MST model) or a tandem set of AD's (DMST model) which do not fit to the curved surface of the VAWT turbine. Therefore, the so-called Actuator Cylinder AC model will be used for modeling the VAWT induction in HAWC2.

Organization of the paper is such that the AC model will be briefly described in Section 2. The derivation of a corrected, linear solution, which is an important version of the AC model due to its low computational requirement, will be shown in Section 3 including results compared with the results of the full model. Finally, the basic principles of implementing the AC model in HAWC2 will be presented in Section 4 as well as an example of simulations on a $5 \mathrm{MW}$ turbine operating in turbulent inflow.

\section{The Actuator Cylinder Flow Model}

The AC model was developed from 1979 to 1982 in a PhD study by Madsen ${ }^{10}$. The basic idea behind the model is to extend the well-known AD flow model concept for HAWT's to a general approach of an actuator surface coinciding with the swept area of the actual turbine ${ }^{10}$. For a straight bladed VAWT the swept surface is cylindrical and this is the surface geometry the model has been developed for in its present form. Further, the model is in a 2D version in order to limit the complexity of the model and thus make it suitable for implementation in an aeroelastic model but the general model is described in a $3 \mathrm{D}$ version ${ }^{11}$.

Following the $\mathrm{AD}$ concept the reaction $Q_{n}(\theta), Q_{t}(\theta)$ of the blade forces $F_{n}(\theta) F_{t}(\theta)$ are in the $\mathrm{AC}$ model applied on the flow as volume or body forces perpendicular and tangential to the rotor plane, respectively, Fig. 3a and Fig. $3 \mathrm{~b}$. The basic equations and a solution method have been presented by Madsen ${ }^{10}$ and will only be briefly described here. 
The basic equations are the Euler equations in $2 \mathrm{D}$ and the velocity components $v_{x}, v_{y}$ are written as:

$$
v_{x}=1+w_{x} \quad \text { and } \quad v_{y}=w_{y}
$$

in order to split the solution to the equations in a linear and a non-linear term. It should be noted that the equations are non-dimensionalized with the basic dimensions $R, V_{\infty}, \rho$ which are rotor radius, free stream velocity and flow density.

Now the Euler Equations take the form:

$$
\begin{aligned}
& \frac{\partial w_{x}}{\partial x}+w_{x} \frac{\partial w_{x}}{\partial x}+w_{y} \frac{\partial w_{x}}{\partial y}=-\frac{\partial p}{\partial x}+f_{x} \\
& \frac{\partial w_{y}}{\partial x}+w_{x} \frac{\partial w_{y}}{\partial x}+w_{y} \frac{\partial w_{y}}{\partial y}=-\frac{\partial p}{\partial y}+f_{y}
\end{aligned}
$$

and the equation of continuity

$$
\frac{\partial w_{x}}{\partial x}+\frac{\partial w_{y}}{\partial y}=0
$$

In Eq. (2) $p$ is pressure and $f$ are volume forces.

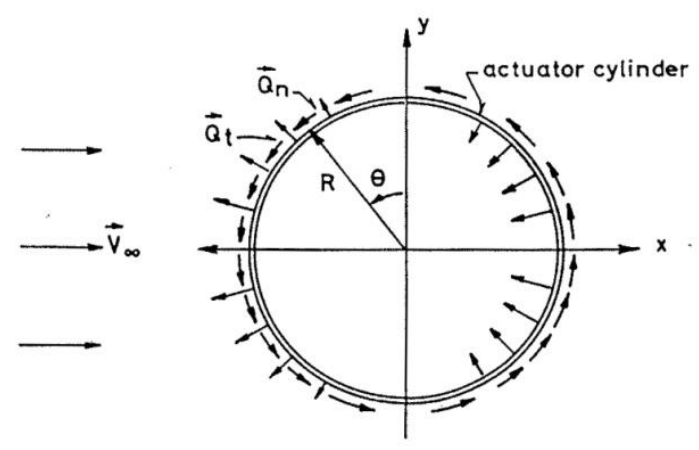

Figure 2a. The Actuator Cylinder flow model representation of a VAWT with volume forces normal and tangential to the circle.

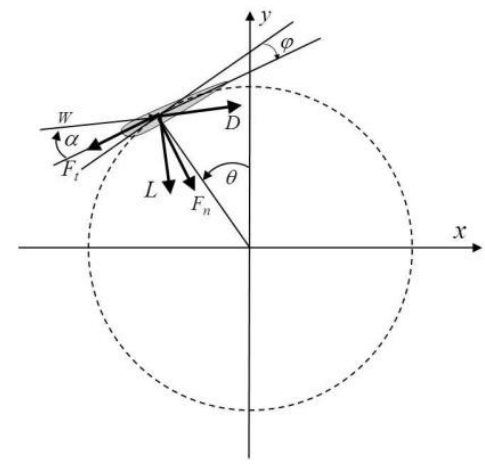

Figure 2b. The blade forces projected on a direction perpendicular and tangential to the circular blade path.

As the volume forces are applied over on an infinitely small radial distance constituting the actuator surface, they are specified by their integral values, $Q_{n}(\theta), Q_{t}(\theta)$ over this infinitely small radial distance and derived from the blade forces per unit blade length by the equations:

$$
\begin{gathered}
Q_{n}(\theta)=\frac{B F_{n}(\theta)}{2 \pi R \rho V_{\infty}^{2}} \\
Q_{t}(\theta)=-\frac{B F_{t}(\theta)}{2 \pi R \rho V_{\infty}^{2}}
\end{gathered}
$$

where $B$ is number of blades and $R$ is the radius of the blade path. 
The Eqs. (2) are now rewritten as:

$$
\begin{aligned}
& \frac{\partial w_{x}}{\partial x}=-\frac{\partial p}{\partial x}+f_{x}+g_{x} \\
& \frac{\partial w_{y}}{\partial x}=-\frac{\partial p}{\partial y}+f_{y}+g_{y}
\end{aligned}
$$

where $g_{x}, g_{y}$ are induced or second order forces defined by:

$$
\begin{aligned}
& g_{x}=-\left(w_{x} \frac{\partial w_{x}}{\partial x}+w_{y} \frac{\partial w_{x}}{\partial y}\right) \\
& g_{y}=-\left(w_{x} \frac{\partial w_{y}}{\partial x}+w_{y} \frac{\partial w_{y}}{\partial y}\right)
\end{aligned}
$$

The following Poisson type equation can then be derived for the pressure:

$$
\frac{\partial^{2} p}{\partial x^{2}}+\frac{\partial^{2} p}{\partial y^{2}}=\left(\frac{\partial f_{x}}{\partial x}+\frac{\partial f_{y}}{\partial y}\right)+\left(\frac{\partial g_{x}}{\partial x}+\frac{\partial g_{y}}{\partial y}\right)
$$

The solution to this equation with the boundary conditions $p \rightarrow 0$ for $x \wedge y \rightarrow \infty$ can be written as:

$$
p(f)=\frac{1}{2 \pi} \iint \frac{f_{x}(x-\xi)+f_{y}(y-\eta)}{(x-\xi)^{2}+(y-\eta)^{2}} d \xi d \eta
$$

and

$$
p(g)=\frac{1}{2 \pi} \iint \frac{g_{x}(x-\xi)+g_{y}(y-\eta)}{(x-\xi)^{2}+(y-\eta)^{2}} d \xi d \eta
$$

The integrals have to be performed throughout the region where the volume forces are different from zero. Once the pressure field has been found the velocities can be derived by integrating Eqs. (6):

$$
\begin{aligned}
& w_{x}=-p(f)+I f_{x}-p(g)+I g_{x}=w_{x}(f)+w_{x}(g) \\
& w_{y}=\int_{-\infty}^{x} \frac{\partial}{\partial y} p(f) d x^{\prime}+I f_{y}-\int_{-\infty}^{x} \frac{\partial}{\partial y} p(g) d x^{\prime}+I g_{y}=w_{y}(f)+w_{y}(g)
\end{aligned}
$$

where

$$
\begin{aligned}
& I f_{x}=\int_{-\infty}^{x} f_{x} d x^{\prime} \text { and } I f_{y}=\int_{-\infty}^{x} f_{y} d x^{\prime} \\
& I g_{x}=\int_{-\infty}^{x} g_{x} d x^{\prime} \text { and } I g_{y}=\int_{-\infty}^{x} g_{y} d x^{\prime}
\end{aligned}
$$


The Eqs. (11) and (12) show that the final solution can be written as a sum of two parts; a linear part which is a function of the prescribed forces $f$ and a non-linear part being function of the induced or second order forces $g$. This is an important characteristic of the solution because the prescribed forces only are applied on a circle and therefore the pressure solution for the linear part becomes the solution of the Laplace equation in two connected regions; outside the cylinder and inside the cylinder, respectively.

\section{A. Linear solution}

For the normal force loading on the $\mathrm{AC}$ which is by far the biggest loading compared with the tangential loading, Figure $3 \mathrm{a}$, the linear solution can be worked out to be ${ }^{10}$ :

$$
\begin{aligned}
& w_{x}=-\frac{1}{2 \pi} \int_{0}^{2 \pi} Q_{n}(\theta) \frac{-(x+\sin (\theta)) \sin (\theta)+(y-\cos (\theta)) \cos (\theta)}{(x+\sin (\theta))^{2}+(y-\cos (\theta))^{2}} d \theta-Q_{n}(\arccos (y))^{*}+Q_{n}(-\arccos (y))^{* *} \\
& w_{y}=-\frac{1}{2 \pi} \int_{0}^{2 \pi} Q_{n}(\theta) \frac{-(x+\sin (\theta)) \cos (\theta)-(y-\cos (\theta)) \sin (\theta)}{(x+\sin (\theta))^{2}+(y-\cos (\theta))^{2}} d \theta
\end{aligned}
$$

The term marked with * in Eq. (15) shall only be added inside the cylinder whereas in the wake behind the cylinder both the term marked with * and ** shall be added.

Assuming that the loading is piece-wise constant we can find the integral part in Eq. (15) and Eq. 16 as:

$$
\begin{aligned}
& w_{x}=\sum_{i=1}^{i=N} Q_{n, i} \int_{\theta_{i}-1 / 2 \Delta \theta}^{\theta_{i}+\frac{1}{2} \Delta \theta} \frac{-(x+\sin (\theta)) \sin (\theta)+(y-\cos (\theta)) \cos (\theta)}{(x+\sin (\theta))^{2}+(y-\cos (\theta))^{2}} d \theta \\
& w_{y}=\sum_{i=1}^{i=N} Q_{n, i} \int_{\theta_{i}-1 / 2 \Delta \theta}^{\theta_{i}+1 / 2 \Delta \theta} \frac{-(x+\sin (\theta)) \cos (\theta)-(y-\cos (\theta)) \sin (\theta)}{(x+\sin (\theta))^{2}+(y-\cos (\theta))^{2}} d \theta
\end{aligned}
$$

In the simulations we have typically used: $\Delta \theta=10 \mathrm{deg} ., N=36$ and $\theta_{i}=5,15,35 \ldots$. deg. etc.

We can now write the total solution to Eqs. (15) and 16 on the blade path as:

$$
w_{x}(j)=\sum_{i=1}^{i=N} Q_{n, i} R_{w_{x}}(i, j)-Q_{n, j}^{*}+Q_{n,(N-j)}^{*}
$$

The star terms are only added for $\mathrm{j}>18$ (the leeward part of the AC).

$$
w_{y}(j)=\sum_{i=1}^{i=N} Q_{n, i} R_{w_{y}}(i, j)
$$

where the influence coefficients are:

$$
R_{w_{x}}(i, j)=\int_{\theta_{i}-1 / 2 \Delta \theta}^{\theta_{i}+1 / 2 \Delta \theta} \frac{-(x(j)+\sin (\theta)) \sin (\theta)+(y(j)-\cos (\theta)) \cos (\theta)}{(x(j)+\sin (\theta))^{2}+(y(j)-\cos (\theta))^{2}} d \theta
$$




$$
R_{w_{y}}(i, j)=\int_{\theta_{i}-1 / 2 \Delta \theta}^{\theta_{i}+1 / 2 \Delta \theta} \frac{-(x(j)+\sin (\theta)) \cos (\theta)-(y(j)-\cos (\theta)) \sin (\theta)}{(x(j)+\sin (\theta))^{2}+(y(j)-\cos (\theta))^{2}} d \theta
$$

and

$$
\begin{array}{ll}
x(j)=-\cos (j \Delta \varphi-1 / 2 \Delta \varphi) & j=1,2 \ldots . .36 \\
y(j)=\sin (j \Delta \varphi-1 / 2 \Delta \varphi) & j=1,2 \ldots \ldots . .36
\end{array}
$$

The influence coefficients in Eqs. (21) and (22) can be integrated once and for all. It means that the linear solution given by Eqs. (19) and (20) only requires a minimum of computing.

\section{B. Non-linear solution}

The non-linear solution is obtained through an iteration following the scheme:

$$
\begin{aligned}
& w_{x}^{n}=w_{x}(f)-p\left(g^{n-1}\right)+I g_{x}^{n-1}=w_{x}(f)+w_{x}\left(g^{n-1}\right) \\
& w_{y}^{n}=w_{y}(f)-\int_{-\infty}^{x} \frac{\partial}{\partial y} p\left(g^{n-1}\right)+I g_{y}^{n-1}=w_{y}(f)+w_{y}\left(g^{n-1}\right)
\end{aligned}
$$

where $w_{x}^{n}, w_{y}^{n}$ is the result of the $\mathrm{n}^{\text {th }}$ iteration. In Ref. 10 a semi analytical/numerical method for solving the Poisson equation for the pressure due to the second order terms is presented but it will not be further elaborated here.

\section{Results}

Computations have been run on a small VAWT used in the DeepWind project for code comparison. The airfoil data have been idealized to a linear lift curve as function of inflow angle with a slope of $2 \pi$ and no stall. The airfoil drag is constant 0.023 . Different solidities have been tested but in the present paper a solidity of 0.1 has been chosen. The solidity $\sigma$ is in this case defined as:

$$
\sigma=\frac{B c}{2 R}
$$

It should further be noted that it is a $2 \mathrm{D}$ computation (no computational slices in vertical direction) and finally that the model in this case is a separate AC numerical model so it does not involve HAWC2.

Results for this case are shown in Figures 3 and 4 and the main objective is to show the difference between the linear model and the full model with the second order forces. In the left part of Fig. 3 is shown the power coefficient and the thrust coefficient as function of tip speed ratio and it is seen that the accuracy of the linear model is only satisfactory up to a tip speed ratio of about 2.5 where the thrust coefficient reaches a value of 0.6 to 0.7 . To the right in Fig. 3 the normal and tangential blade forces are compared at a tip speed ratio of 4 . In general the linear model overestimates both forces. In Fig. 5 the flow velocity (magnitude and flow angle) along the blade path is shown at two tip speed ratios. The comparisons clearly show that the differences in the flow field increase with tip speed ratio. 

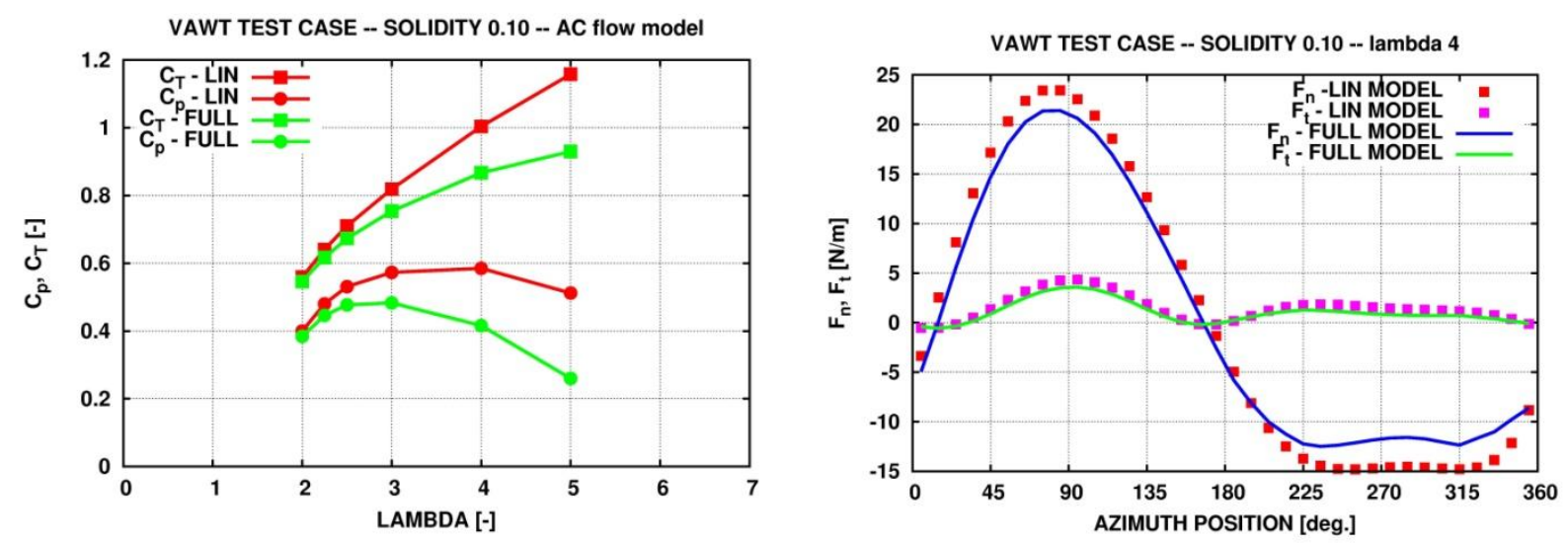

Figure 3. To the left comparison of power coefficient and thrust coefficient computed with the full model and the linear model, respectively. To the right comparison of blade loads at a tip speed ratio of 4.
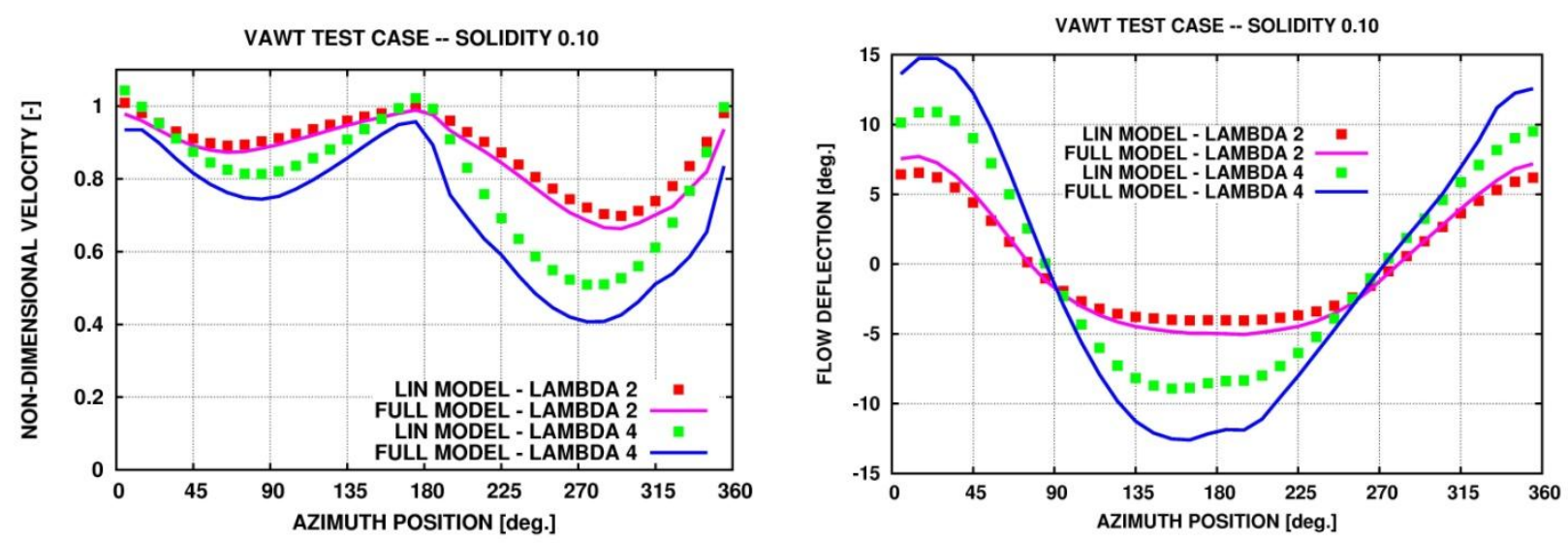

Figure 4. To the left magnitude of velocity along the rotor periphery computed with the full model and the linear model, at two different tip speed ratios, respectively. To the right the flow deflection for the same cases.

\section{A modified linear solution}

Although the full AC model is relative fast (in the range of 30-60 seconds for one wind speed) on a standard pc it means that the model cannot be used to update the flow field at each time step in an aeroelastic simulation. An option could be prior to an aeroelastic time simulation to compute the full flow field at e.g. a few wind speeds around the mean wind speed to be used in the aeroelastic simulation and then derive the instantaneous induction from such data base.

However, it has been investigated if an improved linear model could be derived without increasing the computational demands notable. In Ref. 10 is shown one such modified linear solution where some of the second order terms are included. However, the accuracy of this solution was still too low at high loading.

On the other hand the comparisons of the results from the full model and the linear model, respectively, show that in general there is a good correlation between the shapes of e.g. the curves for the velocities but the induced velocities are too small in the linear model at high loading. This indicates that by amplifying the computed induced velocities from the linear model, a modified linear model with better accuracy might be possible. Such a factor is now derived by looking at the same set of equations and method of solution for the $2 \mathrm{D}$ actuator disc as just derived for the $\mathrm{AC}$ model. The linear solution for the $2 \mathrm{D}$ actuator disc was presented by Madsen ${ }^{11}$ and using the same notation as for the $\mathrm{AC}$ model and the $\mathrm{AD}$ placed at $\mathrm{x}=0$ and stretching from $\mathrm{y}=-1$ to $\mathrm{y}=1$ the solution for the velocities takes the form:

$$
v_{x}=1-\frac{\Delta p}{2 \pi}\left(\operatorname{arctg}\left(\frac{1-y}{x}\right)+\operatorname{arctg}\left(\frac{1+y}{x}\right)\right)-\Delta p^{*}
$$




$$
v_{y}=\frac{\Delta p}{4 \pi} \ln \left(\frac{x^{2}+(y+1)^{2}}{x^{2}+(y-1)^{2}}\right)
$$

Here $\Delta p$ is the pressure drop over the disc and the star means that this term shall only be added in the wake region.

It can now be shown that for a uniform loading this solution gives the following relation between the thrust coefficient $C_{T}$ and the induction $a$ :

$$
C_{T}=4 a_{\text {lin }}
$$

However from the BEM theory we have that:

$$
C_{T}=4 a-4 a^{2}
$$

If we now will achieve the same induction in the linear solution as in the BEM we have to multiply $a_{\text {lin }}$ with the factor:

$$
k_{a}=\frac{1}{1-a}
$$

For the induction factor in Eq. 32 we use a mean induction derived from the thrust coefficient of the whole rotor and using Eq. (31). Multiplying now the velocities from the linear model $w_{x}(f), w_{y}(f)$ with the $k_{a}$ factor we have a model called the MOD LIN model.

\section{Results from the MOD LIN model in comparison with the full model}

Similar figures as presented above for the linear model are shown in Figures 5 and 6 but now with results from the modified model. As can be seen the MOD LIN model results are very close to the full model results and we have therefore now an AC model with a high accuracy and very low computational requirements that can be implemented in an aeroelastic model.
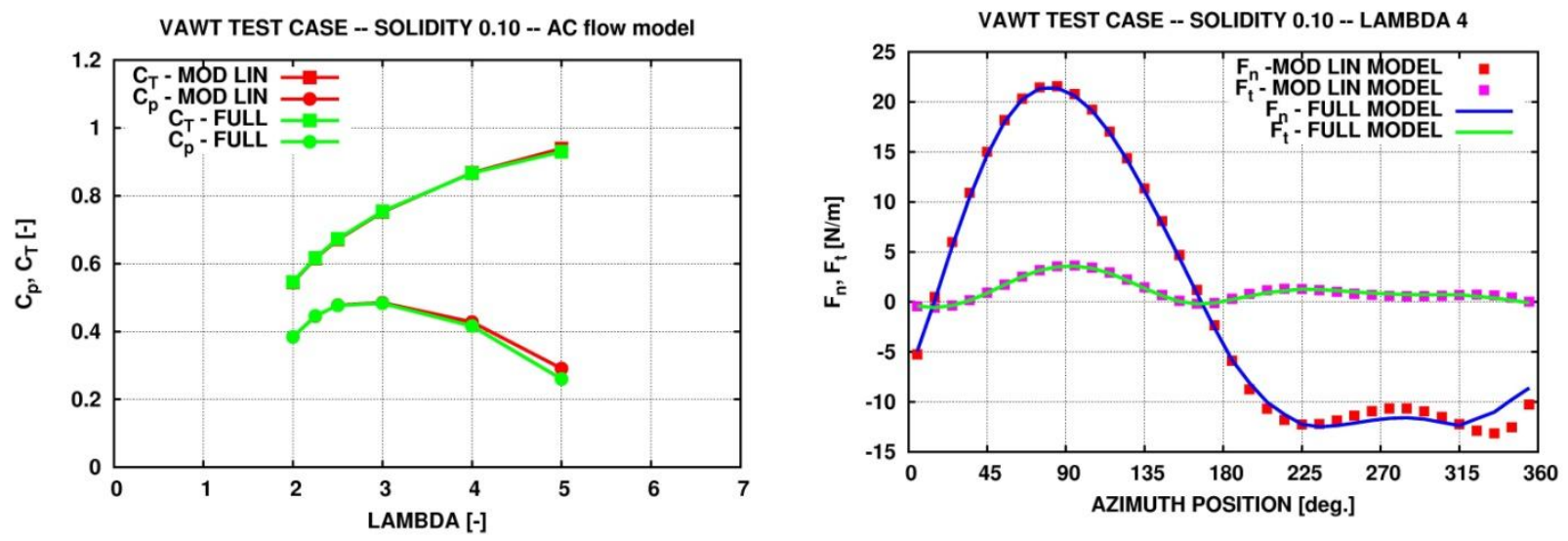

Figure 5. To the left comparison of power coefficient and thrust coefficient computed with the full model and the modified linear model, respectively. To the right comparison of blade loads at a tip speed ratio of 4. 

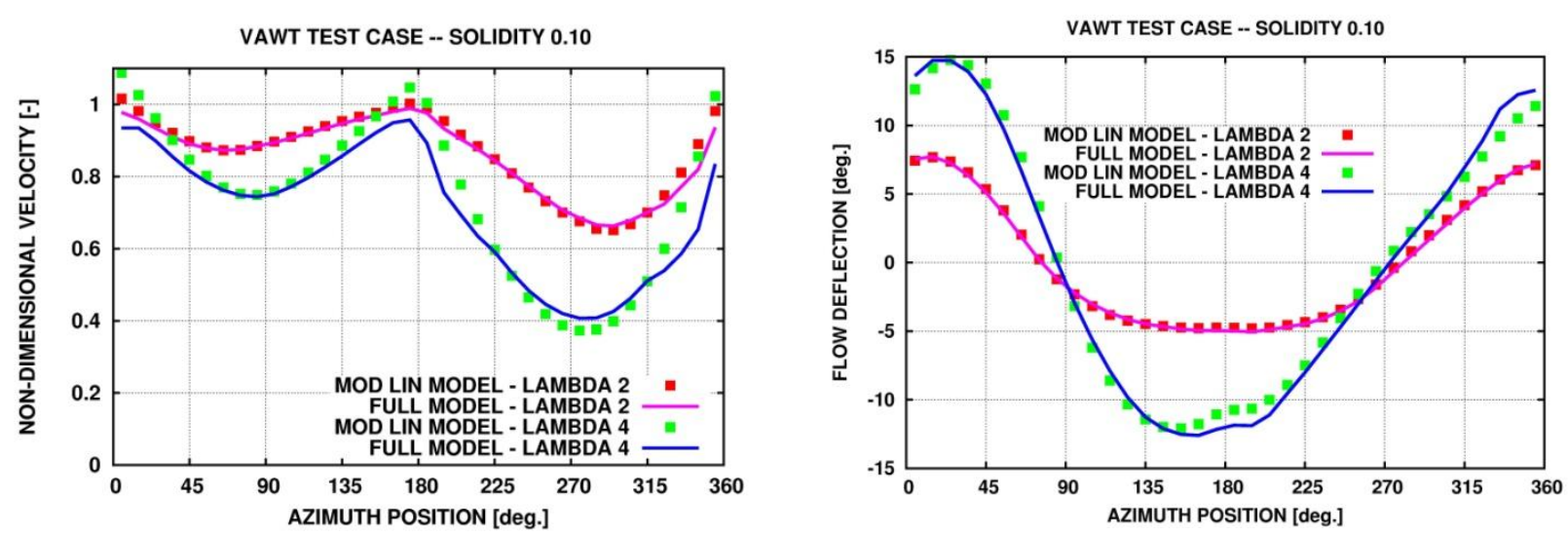

Figure 6. To the left magnitude of velocity along the rotor periphery computed with the full model and the modified linear model, at two different tip speed ratios, respectively. To the right the flow deflection for the same cases.

\section{Implementation of the AC model in HAWC2}

The basic principles in implementing the AC model in HAWC2 are the same as for the HAWT induction modeling in HAWC2. It means that the induction at each time step is computed in a number of points on a grid coinciding with the swept surface of the turbine. The grid can move in space if the turbine rotor does as e.g. is the case of the present floating concept and the orientation of the grid follows the overall wind direction. However, the grid does not rotate with the rotor. As the AC model in its present form is two-dimensional (a cylinder surface) and not coinciding with the swept surface of the Darrieus type rotor in Fig. 1a and 1b, an approximation is made by dividing the swept surface into a number of cylinders with finite height, centered around the shaft and with a radius coinciding with the rotor radius at the actual position along the shaft. It means that the flow expansion in vertical direction is not taken into account. A typical number of 20-30 AC's along the shaft is used and for each AC the induction is computed in 36 azimuthal positions as defined by Eqs. 23 and 24.

At each time step during an aeroelastic time simulation the induction is updated in all of the 36 positions of the 20-30 AC's along the shaft. The input to the flow model is the normal and tangential force coefficients as defined in Eqs. 4 and 5. The blade forces in these equations are derived using local free stream velocities including wind shear and turbulence if present and likewise the local free stream velocity is used to non-dimensionalize the forces. Further the induced velocities from the previous time step are used and the contribution to the velocity triangle from the blades eigen motion is derived by a simple interpolation from the two nearest blades to the considered calculation point.

The induced velocities are then computed with the Eqs. 19 and 20. However, as the flow model is quasy steady we apply next a dynamic inflow filter on the computed velocities and have so far used the same time constants as for the HAWT rotor. A more detailed description of the implementation of the AC model in HAWC2 is presented in Ref. 6.

\section{E. Results from simulations with the AC model implemented in HAWC2}

Within the DeepWind project a first 5MW baseline $\operatorname{rotor}^{11}$ has been designed, Fig. 1b. The overall rotor design parameters are:

$\begin{array}{lll}\circ & \text { rotor radius: } & 63.74 \mathrm{~m} \\ \circ & \text { rotor height: } & 129.56 \mathrm{~m} \\ \circ & \text { blade chord : } & 7.45 \mathrm{~m} \\ \circ & \text { airfoil: } & \text { NACA0018 } \\ \circ & \text { number of blades: } & 2 \\ \circ & \text { solidity: } \sigma=\frac{B c}{2 R} & 0.115 \\ & & \\ \circ & \text { rated power: } & 5000 \mathrm{~kW} \\ \circ & \text { rated speed } & 5.26 \mathrm{rpm} \\ \circ & \text { swept area: } & 10743 \mathrm{~m} 2\end{array}$


An example of a HAWC2 simulation on this turbine with the AC model implemented is presented for a mean wind speed of $8 \mathrm{~m} / \mathrm{s}$, a turbulence intensity of $15 \%$ and a rotational speed of $4.41 \mathrm{rpm}$. It should also be noted that in this case an on-shore version of the turbine without the floater is used.

The induced velocities at two positions upstream and downstream on the swept surface on a position mid between the top and bottom of the rotor are presented in Fig. 7. First it can be noted that the induced axial velocity is biggest on the downstream part of the rotor. It can also be seen that the two axial inductions are quite uncorrelated which is due to considerable spatial separation of the two points causing quiet different turbulence velocities at the two positions. It can further be noted that the induction in lateral direction is considerable outboard on the upstream part of the rotor with direct influence on the inflow angle and thus the loading. An example of blade loading is shown in Fig. 8. The normal force has the characteristic 1p variation for a VAWT causing severe fatigue loading.
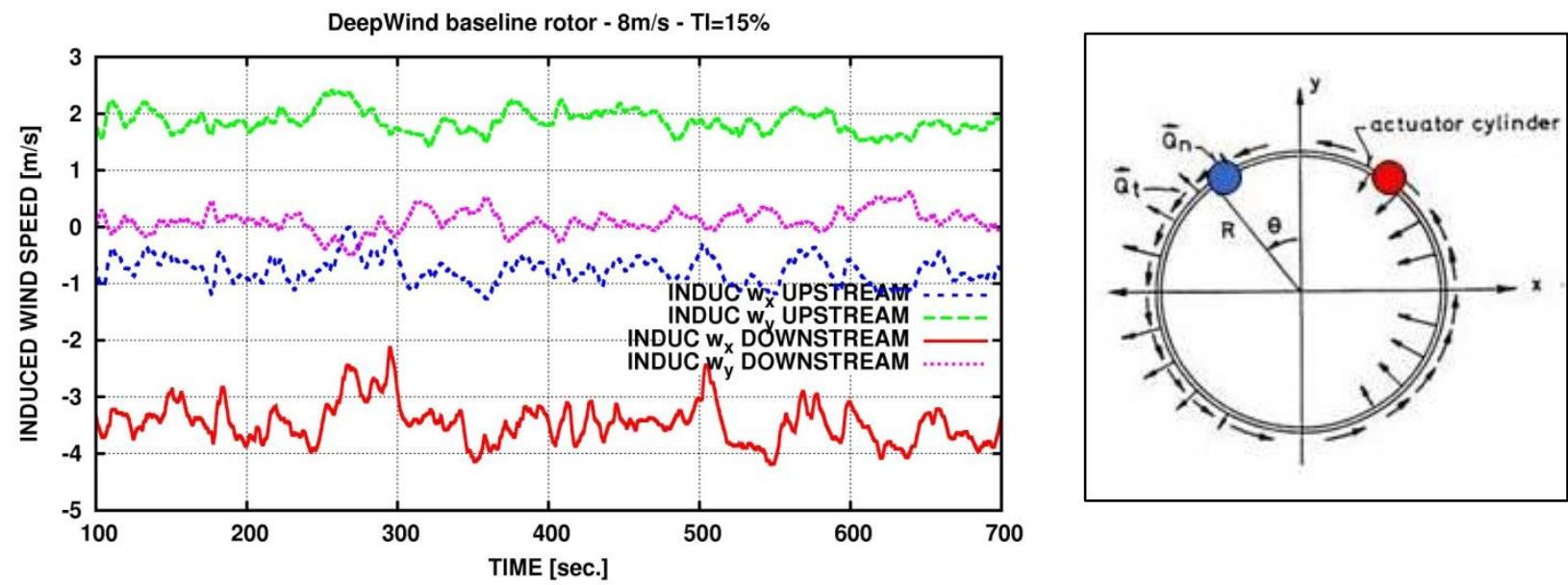

Figure 7. Induced velocities at two positions on the 5MW DeepWind baseline rotor at operation at a mean wind speed of $8 \mathrm{~m} / \mathrm{s}$ and a turbulence intensity of $15 \%$.
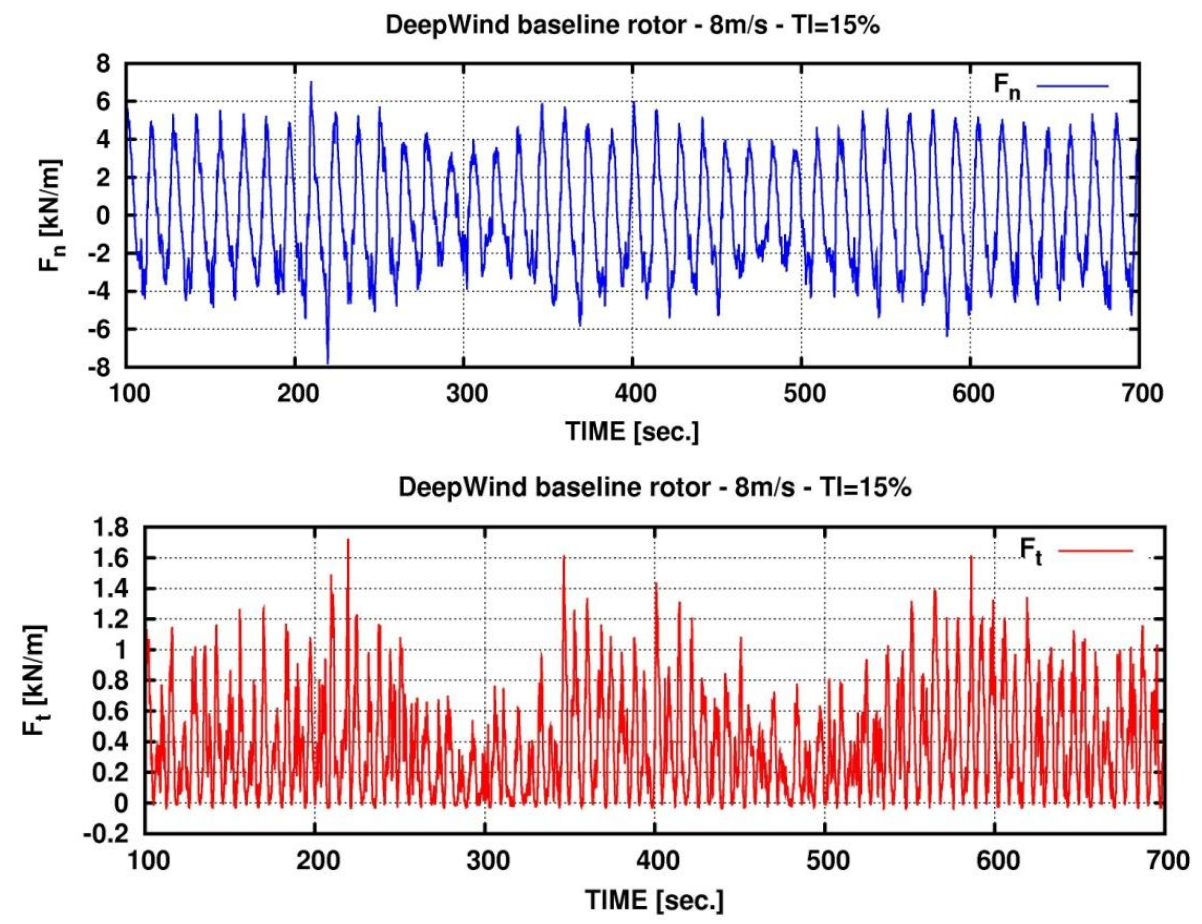

Figure 8. Simulated blade forces in normal and tangential direction at a position of mid between top and bottom of the rotor (rotor height $130 \mathrm{~m})$.

11

American Institute of Aeronautics and Astronautics 
Also the tangential force has a big dynamic content but is only negative during the short periods passing directly against the relative wind.

\section{Conclusion}

The AC flow model for simulation of induction on VAWT's has been presented. In particular, a modified linear MOD LIN version of the model has been derived which gives accurate results even at high turbine loading. The model computation time is very low enabling a detailed computation of the induction over the rotor disc. The AC model has been implemented in the aeroelastic code HAWC2 and typically the induction is updated at each time step in 500-1000 points on a grid coinciding with the rotor swept area. Turbulence in the incoming flow and shear means that the inflow conditions over a MW VAWT rotor varies a lot which requires a detailed computation of the induction.

\section{Acknowledgments}

The DeepWind project is funded by the European Commission, Grant 256769 FP7 Energy 2010- Future emerging technologies.

\section{References}

${ }^{1}$ Vita, L., Paulsen, U. S., Pedersen, T. F., Madsen, H. A., and Rasmussen, F., "A Novel Floating Offshore Wind Turbine Concept," Presented at 2009 European Wind Energy Conference and Exhibition, Marseille, France. Proceedings online 2009, EWEC.

${ }^{2}$ Paulsen, U et al., "DeepWind - an Innovative Wind Turbine Concept for Offshore," Paper presented at EWEA 2011, Brussels, 14-17 March 2011, Belgium. Published by EWEA.

${ }^{3}$ Larsen, T. J., "How 2 Hawc2, the User's Manual,” Ris $\varnothing$ DTU, Roskilde, Denmark 2009.

${ }^{4}$ Vita L., "Offshore Floating Vertical Axis Wind Turbines with Rotating Platform," PhD dissertation PhD 80, Risø DTU, Roskilde, Denmark, 2011.

${ }^{5}$ Vita, L., Paulsen, U. S., Madsen, H. A., Nielsen, P. H., Berthelsen, P. A., Carstensen, S., "Design and Aero-elastic Simulation of a 5MW Floating Vertical Axis Wind Turbine," Proceedings of the ASME 2012 International Conference on Ocean, Offshore and Arctic Engineering OMAE 2012, June 10-June 15, 2012, Rio de Janeiro, Brazil.

${ }^{6}$ Larsen, T. J. and Madsen, H. Aa., "On the way to Reliable Aeroelastic Load Simulation on VAWT's," Paper to be presented at EWEA 2013 in Vienna, Austria from February 4-7, 2013.

${ }^{7}$ Strickland, J. H.. "The Darrieus Turbine: a Performance Prediction Model using Multiple Streamtubes," Albuquerque, Sandia Laboratory, 1975.

${ }^{8}$ Paravischivoiu, I., "Wind Turbine Design, with Emphasis on Darrieus Concept,” Polytechnic International Pres, 2002.

${ }^{9}$ Sutherland, H. J., Berg, D. E., Ashwill, T. D., “A Retrospective of VAWT Technology," SANDIA REPORT SAND20120304 Unlimited Release Printed January 2012.

${ }^{10}$ Madsen, H. A., "The Actuator Cylinder - a Flow Model for Vertical Axis Wind Turbines," PhD thesis, Part 1,2 and 3, Aalborg University Centre, January 1982.

${ }^{11}$ Madsen, H.A. "Application of Actuator Surface Theory on Wind Turbines," Proceedings of IEA meeting on Joint Actions on Aerodynamics of Wind Turbines, 2. Symposium held in Lyngby 21.-22. November 1988, Department of Fluid Mechanics, Technical University of Denmark

${ }^{12}$ Paulsen, U. S., Vita, L., Madsen, H. Aa., Hattel, J., Ritchie, E., Leban, K. M., Berthelsen, P. A., Carstensen, S., "1st DeepWind 5 MW Baseline Design," Proceedings of the DeepSea Conference, 19-20 January 2012, Trondheim, Norway, 2012 Published by Elsevier Ltd. 